\title{
Adding New Perspectives to the Kaleidoscope of Remission Criteria in Rheumatoid Arthritis
}

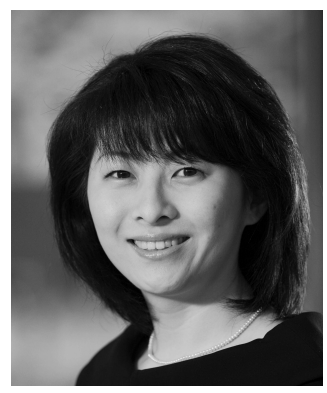

Remission has many meanings. In some medical contexts it indicates lessened disease severity. In other contexts it implies the disease has disappeared. Concepts of remission in rheumatoid arthritis (RA) reflect both models. Some definitions indicate only low disease activity states. Other definitions suggest the absence of disease, with undetectable symptoms, signs, and disease markers. Critically, remission differs from "cure," which implies RA will never return. Current opinion favors restricting remission to patients with either no or minimal synovitis, without longterm structural or functional sequelae. The seminal report by Pinals, et al in 1981 concluded "complete" RA remission indicates the "total absence of articular and extraarticular inflammation and immunological activities" . However, many years later, uncertainties remain on how to define genuine remission states.

The introduction of new therapeutic options and strategies over the past decade has made remission an achievable goal. An immediate consequence of this perspective is the need for an accurate and uniform way to identify remission. Although there are many definitions, remission does not yet have an internationally accepted gold standard. Remission criteria differ between studies, and remission rates vary depending on the remission criteria used. We have previously noted these variations ${ }^{2}$. Some remission criteria use categorical descriptions; the original American College of Rheumatology (ACR) remission criteria are one important example ${ }^{1}$. However, these criteria are very stringent and too few patients achieve this goal to make the definition a useful outcome to discriminate between patients in clinical trial settings, or to make it a realistic outcome in the routine clinic setting. Consequently, many variants have been described. Continuous composite measures are often used to define remission; the most commonly used are the low scores using the Disease Activity Score $\left(\mathrm{DAS}^{3}\right)$ or its modifications such as DAS28-erythrocyte sedimentation rate (DAS-ESR) ${ }^{4}$. The newer criteria include Simplified Disease Activity Index $\left(\mathrm{SDAI}^{5}\right)$, Clinical Disease Activity Index $\left(\mathrm{CDAI}^{6}\right)$, and the ACR/European League Against Rheumatism (ACR/EULAR) Boolean criteria. These aimed to provide a uniform definition that can be widely used ${ }^{7}$. But the Boolean criteria have been criticized as possibly being too stringent. In particular, the patient global visual analog score $>1 \mathrm{~cm}$ (on a $10-\mathrm{cm}$ scale) has proved difficult to achieve ${ }^{8}$. It may not even reflect disease activity as it may relate more to functional limitations, low back pain, and fatigue ${ }^{9}$.

The remission criteria that have evolved over the last 2 decades all reflect a similar underlying theme. Namely, changes in clinical variables assessed by clinicians such as joint counts, physician global scores, and inflammatory blood markers like the ESR or C-reactive protein (CRP). Two reports published in this issue of The Journal add different perspectives to this debate ${ }^{10,11}$. Recognizing that clinicians and patients often have different values and priorities, both articles incorporate outcomes that are important to patients to assess RA disease activity.

The Rheumatoid Arthritis Disease Activity Index-5 (RADAI-5) is a validated patient self-report index. It assesses disease activity, pain, general health, and morning stiffness without capturing detailed self-reported joint counts. Rintelen and colleagues ${ }^{11}$ compared the new ACR/EULAR remission criteria [Boolean and Simplified Disease Activity Index (SDAI) definitions] with remissions defined using the RADAI-5. The cutoff for RADAI-5 remission is 1.4. They studied 705 patients with RA and found that $13 \%$ achieved the ACR/EULAR Boolean-based remission criteria, $24 \%$ achieved SDAI-based remission criteria, and $22 \%$ achieved RADAI-5 remissions. Only $10 \%$ of patients met all 3 criteria. There were significant disparities in the core set variables between RADAI and the comparators (Boolean-based and SDAI-based criteria). Although Health Assessment Questionnaire scores were available in only $60 \%$ of this cohort, where available, scores

See: RA remission criteria instruments, page 386 and 394 
were similar in all 3 remission groups. They found that the SDAI-based definition appeared more sensitive than the RADAI-5 for fulfilling Boolean-based definition of remission (sensitivity $98 \%$ vs $78 \%$ ). Both these criteria had the same specificity and negative predictive values and similar positive predictive values when using Boolean criteria as the reference point. The authors emphasized that a treat-to-target approach should be based on shared decision-making between patient and rheumatologist. Asking patients about their disease activity using RADAI-5 appeared an appropriate means to identify patients' perspectives about their disease.

Castrejon, et $a l^{10}$ have taken a somewhat different approach in defining remission. They combined a patient-reported outcome (PRO) with a physician's assessment by means of a more "relaxed" joint count. They assessed this using the ESPOIR cohort, which is a large prospective French observational study of early RA. They explored 5 different potential criteria for remission in RA. These were based around the Routine Assessment of Patient Index Data 3 (RAPID 3), an index of 3 patient self-report measures - physical function, pain, and patient global estimate of status. They combined RAPID 3 scores with a careful joint examination with or without the physician global estimate. The 5 criteria comprised different combinations of these 3 measures. The Boolean remission criteria and SDAI $<3.3$ were the gold standards applied to the study as well. They argued that if 2 swollen joints are found within a few seconds on a careful joint examination, no one would doubt that the patient was not in remission. In such circumstances, there would be no need for a formal joint count. Although this would be useful to exclude remission, to establish that a patient is in remission with no more than a single swollen joint, a full examination would be required. They found a range of different remission rates for the different remission criteria. These ranged from $33 \%$ of patients meeting the criteria for DAS28 remission, between $13 \%$ and $25 \%$ for the different RAPID 3 remission scores, $18 \%$ for Clinical Disease Activity Index remission, $17 \%$ for SDAI remission, and $13 \%$ for Boolean remission. They found substantial agreement with the Boolean criteria with all the proposed criteria except DAS28 and RAPID 3 remission. Out of the different RAPID 3-based criteria, RAPID 3 remission criteria with 1 swollen joint (RAPID3R+SJ1) was most similar to Boolean and SDAI-based criteria.

Treating RA involves shared decision-making between patients and rheumatologists. It is therefore crucial to include an assessment of patients' perspectives of key outcomes like remission. Using either the RADAI-5 or the RAPID3R+SJ1 to record patients' viewpoints, both provide useful additions to disease assessment in routine clinical practice. Indeed, patient self-assessment has many potential benefits in addition to assessing remission. Self-assessments can be completed while patients are waiting to be seen in the clinic. They can also be undertaken at home and sent to the supervising rheumatologist remotely. Such approaches have the dual benefits of involving patients in their management and optimizing clinical time in focusing on disease management rather than disease assessment within the clinical consultation.

However, self-assessment is not without drawbacks. Although the average patient may be a good judge of their disease, the accuracy of patients' assessments of disease activity and remission may vary. Relying solely on patient perspective may result in suboptimal assessments in some patients. Although blood tests and clinician assessments can be standardized, it is more challenging to standardize patient assessments. A second problem is the diversity of different patient self-assessments. If clinicians move towards greater use of self-assessments, this needs to be accompanied by an agreement to use a limited number of assessments. Adopting a multiplicity of different measures will create major difficulties in comparing outcomes between units or in delivering consistent care when patients move from one center to another.

Despite attempts to define remission in many different forms, the definition of true remission remains elusive. A significant proportion of patients classified in clinical remission continue to develop radiographic progression ${ }^{2,12}$. This suggests ongoing subclinical disease in some patients who are apparently in remission. Radiological markers such as magnetic resonance imaging or ultrasound may be more sensitive in detecting subclinical disease, although there are challenges in standardizing these techniques between clinicians and centers.

Finally, there are 2 outstanding features of remission that need to be clarified. First, there is very little information about how long remission should last to be significant. Meeting criteria for remission on a single occasion is likely to be insufficient. A full clinical picture requires integrating disease states with time. Second, as more patients achieve low disease activity states, there will be more opportunities for drug tapering and withdrawal. Studies are urgently needed to determine which characteristics of remission most accurately predict those patients who are able to withdraw therapy.

In an era where treatments are increasingly targeted at a molecular level, it is important that monitoring treatment responses progresses to reflect such specific therapies. Laboratory biomarkers, beyond ESR and CRP, are likely to play crucial roles in defining disease activity and remission in the future. It is likely that addition of radiological and laboratory biomarkers to clinical variables and patient-reported measures will help clarify the kaleidoscope-like appearance of existing remission criteria. This will give a clearer picture of what true remission entails. 
MARGARET H.Y. MA, MBBS, BSc, MRCP, Department of Rheumatology,

GKT School of Medicine,

Weston Education Centre,

King's College London;

ANDREW P. COPE, BSc, PhD, FRCP;

DAVID L. SCOTT, BSc, MD, FRCP,

Department of Rheumatology,

King's College London,

London, UK

Address correspondence to Dr. H.Y. Ma, Department of Rheumatology, GKT School of Medicine, Weston Education Centre, King's College London, 10 Cutcombe Road, London SE5 9RS, UK.

E-mail: margaret.ma@nhs.net

\section{REFERENCES}

1. Pinals RS, Masi AT, Larsen RA. Preliminary criteria for clinical remission in rheumatoid arthritis. Arthritis Rheum 1981;24:1308-15.

2. Ma MHY, Scott IC, Kingsley GH, Scott DL. Remission in early rheumatoid arthritis. J Rheumatol 2010;37:1444-53.

3. Prevoo ML, van Gestel AM, van 't Hof MA, van Rijswijk MH, van de Putte LB, van Riel PL. Remission in a prospective study of patients with rheumatoid arthritis. American Rheumatism Association preliminary remission criteria in relation to the disease activity score. Br J Rheumatol 1996;35:1101-5.

4. Fransen J, Creemers MCW, van Riel PLCM. Remission in rheumatoid arthritis: Agreement of the Disease Activity Score (DAS28) with the ARA preliminary remission criteria. Rheumatology 2004;43:1252-5.

5. Aletaha D, Ward MM, Machold KP, Nell VP, Stamm T, Smolen JS Remission and active disease in rheumatoid arthritis: Defining criteria for disease activity states. Arthritis Rheum 2005;52:2625-36.
6. Aletaha D, Nell VP, Stamm T, Uffman M, Plufbeil S, Machold K, et al. Acute phase reactants add little to composite disease activity indices for rheumatoid arthritis: Validation of a clinical activity score. Arthritis Res Ther 2005;7:R796-806.

7. Felson DT, Smolen JS, Wells G, Zhang B, van Tuyl LH, Funovits J, et al. American College of Rheumatology/European League Against Rheumatism provisional definition of remission in rheumatoid arthritis for clinical trials. Arthritis Rheum 2011;63:573-86.

8. Studenic P, Smolen JS, Aletaha D. Near misses of ACR/EULAR criteria for remission: Effects of patient global assessment in Boolean and index-based definitions. Ann Rheum Dis 2012;71:1702-5.

9. Masri KR, Shaver TS, Shahouri SH, Wang S, Anderson JD, Busch $\mathrm{RE}$, et al. Validity and reliability problems with patient global as a component of the ACR/EULAR remission criteria as used in clinical practice. J Rheumatol 2012;39:1139-45.

10. Castrejón I, Dougados M, Combe B, Guillemin F, Fautrel B, Pincus T. Can remission in rheumatoid arthritis be assessed without laboratory tests or a formal joint count? Possible remission criteria based on a self-report RAPID 3 score and careful joint examination in the ESPOIR cohort. J Rheumatol 2013;40:386-93.

11. Rintelen B, Sautner J, Haindl B, Mai M, Brezinschek HP, Leeb B. Remission in rheumatoid arthritis: A comparison of the 2 newly proposed ACR/EULAR remission criteria with the Rheumatoid Arthritis Disease Activity Index-5, a patient self-report disease activity index. J Rheumatol 2013;40:394-400.

12. Brown AK, Conaghan PG, Karim Z, Quinn MA, Ikeda K, Peterfy $\mathrm{CG}$, et al. An explanation for the apparent dissociation between clinical remission and continued structural deterioration in rheumatoid arthritis. Arthritis Rheum 2008;58:2958-67.

J Rheumatol 2013;40:353-5; doi:10.3899/jrheum.130099 